\title{
Minimal Total Dominating Functions of Corona Product Graph of a Cycle with a Complete Graph
}

\author{
M.Siva Parvathi \\ Dept. of Mathematics, K.R.K. Govt. Degree \\ College, Addanki-523201, Andhra Pradesh, India
}

\author{
B.Maheswari \\ Dept. of Applied Mathematics, S.P.Women's \\ University, Tirupat-517502, Andhra Pradesh, India
}

\begin{abstract}
Domination in graphs is the fast growing area of research in Graph theory that has emerged rapidly in the last three decades. An introduction and an extensive overview on domination in graphs and related topics is surveyed and detailed in the two books by Haynes et al. [ 6,7 ]. They have applications in diverse areas such as logistics and networks design, mobile computing, resource allocation and telecommunication etc.

Product of graphs occurs naturally in discrete mathematics as tools in combinatorial constructions. They give rise to important classes of graphs and deep structural problems. In this paper the concept of total dominating functions of corona product graph of a cycle with a complete graph is studied and some results on minimal total dominating functions are obtained.
\end{abstract}

Key Words: Corona Product, Cycle, Complete Graph, Total dominating function,

Subject Classification: 68R10

\section{INTRODUCTION}

Domination Theory is an important branch of Graph Theory that has many applications in Engineering, Communication Networks and many others. Allan, R.B. and Laskar, R.[1], Cockayne, E.J. and Hedetniemi, S.T. [2] have studied various domination parameters of graphs.

Recently, dominating functions in domination theory have received much attention. The concepts of total dominating functions and minimal total dominating functions are introduced by Cockayne et al. [3]. Jeelani Begum, S. [8] has studied some total dominating functions of Quadratic Residue Cayley graphs.

Frucht and Harary [5] introduced a new product on two graphs $G_{1}$ and $G_{2}$, called corona product denoted by $\mathrm{G}_{1} \odot \mathrm{G}_{2}$. The object is to construct a new and simple operation on two graphs $G_{1}$ and $G_{2}$ called their corona, with the property that the group of the new graph is in general isomorphic with the wreath product of the groups of $G_{1}$ and of $G_{2}$.

The authors have studied some dominating functions of corona product graph of a cycle with a complete graph [9] and published papers on minimal dominating functions, some variations of $\mathrm{Y}$ - dominating functions and $\mathrm{Y}-$ total dominating functions $[10,11,12]$.

In this paper the concept of total dominating functions of corona product graph of a cycle with a complete graph is considered and some results on minimal total dominating functions are obtained.

\section{CORONA PRODUCT OF $c_{n}$ AND $\boldsymbol{K}_{m}$}

The corona product of a cycle $C_{\mathrm{n}}$ with a complete graph $K_{\mathrm{m}}$ is a graph obtained by taking one copy of a $\mathrm{n}$ - vertex graph $C_{\mathrm{n}}$ and $\mathrm{n}$ copies of $K_{\mathrm{m}}$ and then joining the $i^{\text {th }}$ vertex of $C_{\mathrm{n}}$ to every vertex of $i^{\text {th }}$ copy of $K_{\mathrm{m}}$ and it is denoted by $C_{\mathrm{n}} \odot K_{\mathrm{ng}}$

\section{TOTAL DOMINATING SETS AND TOTAL DOMINATING FUNCTIONS}

The concepts of total dominating functions and minimal total dominating functions are introduced by Cockayne et al. [4]. In this section some results related to total dominating functions of the graph $G=C_{\mathrm{n}} \odot K_{\mathrm{x}}$ are proved.

Definition: Let $G\left(V_{x} E\right)$ be a graph without isolated vertices. A subset $T$ of $V$ is called a total dominating set (TDS) if every vertex in $V$ is adjacent to at least one vertex in $T$.

If no proper subset of $T$ is a total dominating set, then $T$ is called a minimal total dominating set (MTDS) of $G$.

Definition: The minimum cardinality of a MTDS of $G$ is called a total domination number of $G$ and is denoted by $V_{t}(G)$.

Definition: Let $G\left(V_{v} E\right)$ be a graph. A function $f: V \rightarrow[0,1]$ is called a total dominating function (TDF) of $G$ if $f(W(v))=\sum_{u \in N(v)} f(u) \geq 1$, for each $w \in V$. Here

$\mathbb{N}(\mathbb{v})$ is a open neighbourhood set of $\mathbb{v} \in V$.

Definition: Let $f$ and $g$ be functions from $V$ to $\left[0_{w} 1\right]$. We define $f<g$ if $f(u) \leq g(w)$ for all $u \in V$, with strict inequality for at least one vertex $w \in V$.

A TDF $f$ of $G$ is called a minimal total dominating function (MTDF) if for all $\quad g<f, g$ is not a TDF.

Theorem 3.1: The total domination number of $G=C_{\mathrm{n}} \odot K_{\mathrm{g}}$ is $\mathrm{n}$. 
Proof: Let $T$ denote a total dominating set of $G$. Suppose T contains the vertices of $C_{\mathrm{K}}$.

By the definition of the graph $G$, every vertex in $C_{\mathrm{n}}$ is adjacent to all vertices of associated copy of $K_{\mathrm{m}}$. That is the vertices in $C_{\mathrm{g}}$ dominate the vertices in all copies of $K_{\mathrm{m}}$ respectively. Further these vertices being in $C_{\mathrm{m}}$, they dominate among themselves. Thus $T$ becomes a TDS of $G$.

Therefore $\gamma_{t}(G)=n$.

Theorem 3.2: Let $T$ be a MTDS of $G=C_{\mathrm{n}} \odot K_{\mathrm{m}}$ whose vertex set is $V$. Then a function $f: V \rightarrow\left[0_{w} 1\right]$ defined by

$f(v)= \begin{cases}1, & \text { if } v \in T, \\ 0, & \text { otherwise. }\end{cases}$

becomes a MTDF of $G=C_{\mathrm{g}} \odot K_{\mathrm{g}}$

Proof: Let $T$ be a MTDS of $G$. Obviously this set contains all the vertices of $C_{\mathrm{n}}$ and this set is also minimum.

Case 1: Let $\mathbb{E} \in C_{\mathrm{m}}$ be such that $d(\mathbb{D})=m+2$ in $G$.

Then $\mathbb{N}(\mathbb{D})$ contains $m$ vertices of $K_{\mathrm{g}}$ and two vertices of $C_{\mathrm{n}}$ in $G$.

So $\sum_{u \in N(v)} f(u)=1+1+\underbrace{0+\ldots \ldots+0}_{m \text {-times }}=2$.

Case 2: Let $\mathbb{E} \in K_{\mathrm{m}}$ be such that $d(\mathbb{v})=m$ in $G$.

Then $N(\mathbb{E})$ contains $(m-1)$ vertices of $K_{\mathrm{m}}$ and one vertex of $C_{\mathrm{M}}$ in $G$.

So $\sum_{u \in N(v)} f(u)=1+\underbrace{0+\ldots \ldots+0}_{(m-1)-\text { times }}=1$.

Therefore for all possibilities,

we get $\sum_{u \in N(v)} f(u) \geq 1, \quad$ षै $\mathbb{v} \in \mathbb{V}$.

This implies that $f$ is a TDF.

Now we check for the minimality of $f$.

Define $g: V \rightarrow\left[0_{w} 1\right]$ by

$g(v)= \begin{cases}\mathrm{r}, & \text { if } \mathrm{v}=\mathrm{v}_{\mathrm{k}} \in T, \\ 1, & \text { if } \mathrm{v} \in \mathrm{T}-\left\{\mathrm{v}_{\mathrm{k}}\right\}, \\ 0, & \text { otherwise. }\end{cases}$

where $0<x<1$.

Since strict inequality holds at the vertex $v_{k} \in T$, it follows that $g<f$.

Case 1: Let $v \in C_{n}$ be such that $d(v)=m+2$ in $G$.

Sub case 1: Let $v_{k} \in N(v)$.

Then $\sum_{u \in N(v)} g(u)=r+1+\underbrace{0+\ldots \ldots+0}_{m \text {-times }}=r+1>1$.
Sub case 2: Let $v_{k} \notin N(v)$.

Then $\sum_{u \in N(v)} g(u)=1+1+\underbrace{0+\ldots \ldots+0}_{m \text {-times }}=2$.

Case 2: Let $\mathbb{D} \in K_{\mathrm{m}}$ be such that $d(\mathbb{v})=\mathrm{m}$ in $G$.

Sub case 1: Let $v_{k} \in N(v)$.

Then $\sum_{u \in N(v)} g(u)=r+\underbrace{0+\ldots \ldots .+0}_{(m-1) \text {-times }}=r<1$.

Sub case 2: Let $v_{k} \notin N(v)$.

Then $\sum_{u \in N(v)} g(u)=1+\underbrace{0+\ldots \ldots .+0}_{(m-1) \text {-times }}=1$.

This implies that $\sum_{u \in N(v)} g(u)<1$, for some $\mathrm{v} \in \mathrm{V}$.

So $g$ is not a TDF.

Since $g$ is taken arbitrarily, it follows that there exists no $g<f$ such that $g$ is a TDF.

Thus $f$ is a MTDF.

Theorem 3.3: A function $f: V \rightarrow\left[0_{w} 1\right]$ defined by $f(\mathbb{D})=\frac{1}{q^{\infty}} \forall \mathbb{V} \in \mathbb{V}$ is a TDF of $G=C_{\mathrm{n}} \odot K_{\mathrm{m}}$ if $q \leq m$. It is a MTDF if $q=m$

Proof: Let $f$ be a function defined as in the hypothesis.

Case I: Suppose $0<q<m$.

Case 1: Let $\mathbb{D} \in C_{\mathrm{n}}$ be such that $d(\mathbb{D})=m+2$ in $G$.

Then $\sum_{u \in N(v)} f(u)=\underbrace{\frac{1}{q}+\frac{1}{q}+\ldots \ldots+\frac{1}{q}}_{(m+2)-\text { times }}=\frac{m+2}{q}$.

Since $q<m$, it follows that $\frac{m+2}{q}>1$.

Case 2: Let $\mathbb{D} \in K_{\mathrm{m}}$ be such that $d(\mathbb{D})=m$ in $G$.

Then $\sum_{u \in N(v)} f(u)=\underbrace{\frac{1}{q}+\frac{1}{q}+\ldots \ldots . .+\frac{1}{q}}_{m \text {-times }}=\frac{m}{q}>1$,

since $q<m$

Therefore for all possibilities, we get

$\sum_{u \in N(v)} f(u)>1, \quad \forall \forall \in \mathbb{W}$.

This implies that $f$ is a TDF.

Now we check for the minimality of $f$.

Define $g: V \rightarrow\left[0_{0} 1\right]$ by 
$g(v)= \begin{cases}r, & \text { if } v=v_{k} \in V, \\ \frac{1}{q}, & \text { otherwise. }\end{cases}$

where $0<8<\frac{1}{q}$.

Since strict inequality holds at a vertex $\mathbb{V}_{k}$ of $V$, it follows that $g<f$.

Case (i): Let $\mathbb{D} \in C_{\mathrm{n}}$ be such that $d(\mathbb{D})=m+2$ in $G$.

Sub case 1: Let $v_{k} \in N(v)$.

$$
\text { Then } \begin{aligned}
\sum_{u \in N(v)} g(u) & =r+\underbrace{\frac{1}{q}+\frac{1}{q}+\ldots \ldots .+\frac{1}{q}}_{(m+1) \text {-times }} \\
& <\frac{1}{q}+\frac{m+1}{q}=\frac{m+2}{q}>1, \text { since } \mathrm{q}<m
\end{aligned}
$$

Case 2: Let $v_{k} \notin N(v)$.

Then

$$
\sum_{u \in N(v)} g(u)=\underbrace{\frac{1}{q}+\frac{1}{q}+\ldots \ldots . .+\frac{1}{q}}_{(m+2)-\text { times }}=\frac{m+2}{q}>1,
$$

since $\mathrm{q}<m$

Case (ii): Let $\mathbb{v} \in K_{\mathrm{m}}$ be such that $d(\mathbb{D})=m$ in $G$.

Sub case 1: Let $v_{k} \in N(v)$.

$$
\text { Then } \begin{aligned}
\sum_{u \in N(v)} g(u)=r & +\underbrace{\frac{1}{q}+\frac{1}{q}+\ldots \ldots .+\frac{1}{q}}_{(m-1) \text {-times }} \\
& <\frac{1}{q}+\frac{m-1}{q} \\
& =\frac{m}{q}>1, \quad \text { since } \mathrm{q}<\mathrm{m} .
\end{aligned}
$$

Sub case 2: Let $v_{k} \notin N(v)$. Then

$$
\begin{aligned}
\sum_{u \in N(v)} g(u) & =\underbrace{\frac{1}{q}+\frac{1}{q}+\ldots \ldots . .+\frac{1}{q}}_{m \text {-times }} \\
& =\frac{m}{q}>1 .
\end{aligned}
$$

Hence, it follows that $\sum_{u \in N(v)} g(u)>1$, $\forall \in \mathbb{W}$.

Thus $g$ is a TDF.

This implies that $f$ is not a MTDF.

Case II: Suppose $q=m$.

Substituting $q=m$ in case 1 and 2 , we get

$$
\begin{aligned}
\sum_{u \in N(v)} f(u) & =\underbrace{\frac{1}{q}+\frac{1}{q}+\ldots \ldots .+\frac{1}{q}}_{(m+2)-\text { times }}=\frac{m+2}{q} \\
& =\frac{m+2}{m}=1+\frac{2}{m}>1 .
\end{aligned}
$$

and

$$
\sum_{u \in N(v)} f(u)=\underbrace{\frac{1}{q}+\frac{1}{q}+\ldots \ldots+\frac{1}{q}}_{m \text {-times }}=\frac{m}{q}=\frac{m}{m}=1 .
$$

Therefore for all possibilities, we get

$$
\sum_{u \in N(v)} f(u) \geq 1, \quad \text { षึ } \mathbb{v} \in \mathbb{V}_{a}
$$

This implies that $f$ is a TDF.

Now we check for the minimality of $f$.

Define $g: V \rightarrow[0,1]$ by

$g(v)= \begin{cases}r, & \text { if } v=v_{k} \in V, \\ \frac{1}{q}, & \text { otherwise. }\end{cases}$

where $0<7<\frac{1}{q}$.

Since strict inequality holds at a vertex $\mathbb{V}_{k}$ of $V$, it follows that $g<f$.

We can show as in case (i) of case I that for $\mathbb{E} \in C_{\mathrm{Y}}$ be such that $d(\mathbb{v})=m+2$,

$$
\sum_{u \in N(v)} g(u)=r+\underbrace{\frac{1}{q}+\frac{1}{q}+\ldots \ldots+\frac{1}{q}}_{(m+1)-\text { times }}>1,
$$

if $v_{k} \in N(v)$ and

$$
\sum_{u \in N(v)} g(u)=\underbrace{\frac{1}{q}+\frac{1}{q}+\ldots \ldots .+\frac{1}{q}}_{(m+2) \text {-times }}>1 \text {, if } v_{k} \notin N(v) .
$$

Again as in case (ii) of case I that for $\mathbb{E} \in K_{\mathrm{m}}$ be such that $d(v)=m$, we have

$$
\begin{aligned}
\sum_{u \in N(v)} g(u)= & r+\underbrace{\frac{1}{q}+\frac{1}{q}+\ldots \ldots . .+\frac{1}{q}}_{(m-1)-\text { times }} \\
& <\frac{1}{q}+\frac{m-1}{q}=\frac{m}{m}=1,
\end{aligned}
$$

if $v_{k} \in N(v)$ and

$$
\begin{aligned}
& \sum_{u \in N(v)} g(u)=\underbrace{\frac{1}{q}+\frac{1}{q}+\ldots \ldots . .+\frac{1}{q}}_{m \text {-times }}=\frac{m}{q}=\frac{m}{m}=1, \\
& \text { if } v_{k} \notin N(v) .
\end{aligned}
$$


This implies that $\sum_{u \in N(v)} g(u)<1$, for some $\mathrm{v} \in \mathrm{V}$.

So $g$ is not a TDF.

Since $g$ is defined arbitrarily, it follows that there exists no $g<f$ such that $g$ is a TDF.

Thus $f$ is a MTDF.

Theorem 3.4: A function $f: V \rightarrow\left[0_{w} 1\right]$ defined by $f(\mathbb{v})=\frac{p}{q} \forall \forall \in \mathbb{V}$ where $p=\min (m, n)$ and

$q=\max \left(m_{0} n\right)$ is a TDF of $G=C_{n} \odot K_{\mathrm{m}}$ if $\frac{\mathrm{p}}{q} \geq \frac{1}{\mathrm{~m}}$. Otherwise it is not a TDF. Also it becomes a MTDF if $\frac{p}{q}=\frac{1}{\mathrm{x}}$.

Proof: Let $f: V \rightarrow[0,1]$ be defined by $f(v)=\frac{p}{q}, \forall \forall \in V$, where $p=\min (m, n)$ and $q=\max (m, m)$,

Clearly $\frac{p}{q}>0$.

Case 1: Let $\mathbb{v} \in C_{\mathrm{n}}$ be such that $d(\mathbb{v})=m+2$ in $G$.

Then $\sum_{u \in N(v)} f(u)=\underbrace{\frac{p}{q}+\frac{p}{q}+\ldots \ldots+\frac{p}{q}}_{(m+2) \text {-times }}=(m+2) \frac{p}{q}$.

Case 2: Let $\mathbb{D} \in K_{\mathrm{m}}$ be such that $d(\mathbb{D})=m$ in $G_{x}$ Then

$\sum_{u \in N(v)} f(u)=\underbrace{\frac{p}{q}+\frac{p}{q}+\ldots \ldots . .+\frac{p}{q}}_{m \text {-times }}=m \frac{p}{q}$.

From the above two cases, we observe that $f$ is a TDF if $\frac{p}{q} \geq \frac{1}{x}$.

Otherwise $f$ is not a TDF.

Case 3: Suppose $\frac{\mathrm{p}}{\mathrm{q}}>\frac{1}{\mathrm{~m}}$.

Clearly $f$ is a TDF.

Now we check for the minimality of $f$.

Define $g: V \rightarrow\left[0_{w} 1\right]$ by

$g(v)= \begin{cases}r, & \text { if } v=v_{k} \in V, \\ \frac{p}{q}, & \text { otherwise. }\end{cases}$

where $0<\pi<\frac{p}{Q}$.

Since strict inequality holds at a vertex $\mathbb{V}_{k}$ of $V$, it follows that $g<f$.

Case (i): Let $\mathbb{D} \in C_{m}$ be such that $d(\mathbb{D})=m+2$ in $G$.

Sub case 1: Let $v_{k} \in N(v)$.

Then $\sum_{u \in N(v)} g(u)=r+\underbrace{\frac{p}{q}+\frac{p}{q}+\ldots \ldots+\frac{p}{q}}_{(m+1) \text {-times }}$ since $\frac{p}{q}>\frac{1}{\mathrm{~m}}$.

$$
<\frac{p}{q}+(m+1) \frac{p}{q}=(m+2) \frac{p}{q}>1
$$

Sub case 2: Let $v_{k} \notin N(v)$. Then

$$
\sum_{u \in N(v)} g(u)=\underbrace{\frac{p}{q}+\frac{p}{q}+\ldots \ldots .+\frac{p}{q}}_{(m+2)-\text { times }}=(m+2) \frac{p}{q}>1 .
$$

Case (ii): Let $\mathbb{v} \in K_{\mathrm{m}}$ be such that $d(\mathbb{D})=m$ in $G$.

Sub Case 1: Let $v_{k} \in N(v)$.

$$
\text { Then } \begin{aligned}
\sum_{u \in N(v)} g(u)=r & +\underbrace{\frac{p}{q}+\frac{p}{q}+\ldots \ldots+\frac{p}{q}}_{(m-1)-\text { times }} \\
& <\frac{p}{q}+(m-1) \frac{p}{q}=m \frac{p}{q}>1,
\end{aligned}
$$

since $\frac{p}{q}>\frac{1}{m}$.

Sub case 2: Let $v_{k} \notin N(v)$.

Then

$$
\sum_{u \in N(v)} g(u)=\underbrace{\frac{p}{q}+\frac{p}{q}+\ldots \ldots .+\frac{p}{q}}_{m \text {-times }}=m \frac{p}{q}>1 .
$$

It follows that $\sum_{u \in N(v)} g(u)>1, \mathbb{W} \quad \mathbb{W} \in \mathbb{W}$.

Thus $g$ is a TDF.

This implies that $f$ is not a MTDF.

Case 4: Suppose $\frac{p}{q}=\frac{1}{m}$.

As in case 1 and 2, we have that

$$
\begin{aligned}
\sum_{u \in N(v)} f(u) & =\underbrace{\frac{p}{q}+\frac{p}{q}+\ldots \ldots . .+\frac{p}{q}}_{(m+2)-\text { times }}=(m+2) \frac{p}{q} \\
& =(m+2) \frac{1}{m}=1+\frac{2}{m}>1,
\end{aligned}
$$

if $\mathbb{D} \in \mathcal{C}_{\mathrm{g}}$ and

$$
\sum_{u \in N(v)} f(u)=\underbrace{\frac{p}{q}+\frac{p}{q}+\ldots \ldots+\frac{p}{q}}_{(m) \text {-times }}=m \frac{p}{q}=m \frac{1}{m}=1,
$$

if $\mathbb{D} \in K_{\mathrm{m}}$.

Therefore for all possibilities, we get

$$
\sum_{u \in N(v)} f(u) \geq 1, \mathbb{v} \quad v \in \mathbb{V}
$$

This implies that $f$ is a TDF.

Now we check for the minimality of $f$.

Define $g: V \rightarrow\left[0_{w} 1\right]$ by

$g(v)= \begin{cases}\mathrm{r}, & \text { if } \mathrm{v}=\mathrm{v}_{\mathrm{k}} \in V, \\ \frac{\mathrm{p}}{\mathrm{q}}, & \text { otherwise. }\end{cases}$

where $0<7<\frac{p}{q}$. 
Since strict inequality holds at a vertex $\mathbb{V}_{\mathrm{k}}$ of $\mathbb{V}$, it follows that $g<f$.

Then we can show as in case (i) of case 3 that

$$
\begin{gathered}
\sum_{u \in N(v)} g(u)=r+\underbrace{\frac{p}{q}+\frac{p}{q}+\ldots \ldots+\frac{p}{q}}_{(m+1)-\text { times }}>1, \\
\text { iff } \mathrm{v} \in \mathbb{C}_{\mathrm{n}} \text { and } v_{\mathrm{k}} \in \mathbb{N}(v) .
\end{gathered}
$$

$$
\text { And } \begin{aligned}
& \sum_{u \in N(v)} g(u)=\underbrace{\frac{p}{q}+\frac{p}{q}+\ldots \ldots .+\frac{p}{q}}_{(m+2)-\text { times }}>1, \\
& \text { if } \mathrm{v} \in \mathbb{C}_{\mathrm{n}} \text { and } \mathbb{V}_{k} \in \mathbb{N}(\mathbb{v})_{x}
\end{aligned}
$$

Again as in case (ii) of case 3 , we can show that

$$
\begin{aligned}
& \sum_{u \in N(v)} g(u)=r+\underbrace{\frac{p}{q}+\frac{p}{q}+\ldots \ldots+\frac{p}{q}}_{(m-1) \text {-times }}<1, \\
& \text { if } w \in K_{\mathrm{m}} \text { and } w_{\mathrm{k}} \in \mathrm{N}(\mathrm{v}) \text {, } \\
& \text { And } \sum_{u \in N(v)} g(u)=\underbrace{\frac{p}{q}+\frac{p}{q}+\ldots \ldots .+\frac{p}{q}}_{m \text {-times }}=(m) \frac{p}{q}=(m) \frac{1}{m}=1 \text {, }
\end{aligned}
$$

This implies that $\sum_{u \in N(v)} g(u)<1$, for some $\mathrm{v} \in \mathrm{V}$.

So $g$ is not a TDF.

Since $g$ is defined arbitrarily, it follows that there exists no $g<f$ such that $g$ is a TDF.

Thus $f$ is a MTDF.

\section{CONCLUSION}

It is interesting to study the total dominating functions of the corona product graph of a cycle with a complete graph. This work gives the scope for the study of convexity of these minimal total dominating functions and the authors have also studied this concept.

\section{ILLUSTRATION}

\section{Theorem 3.1}

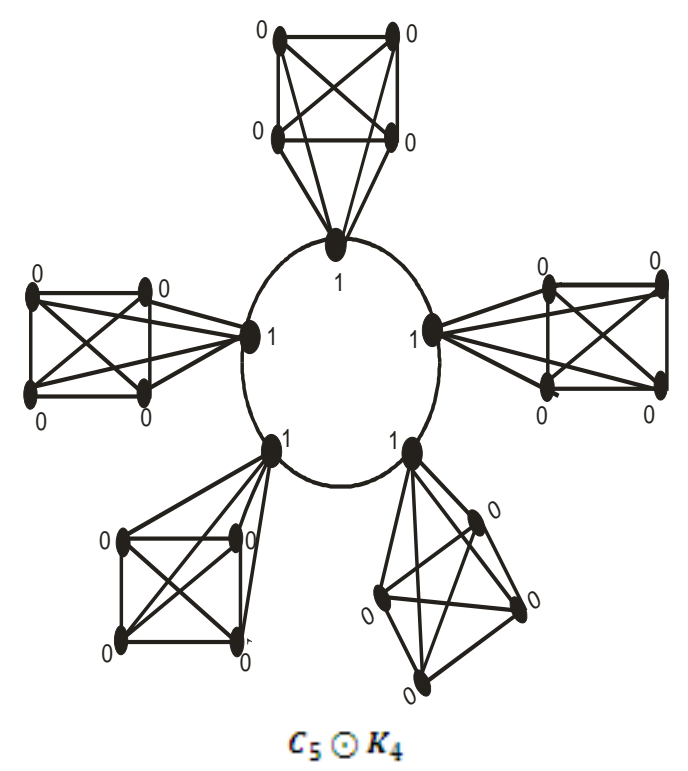

The function $f$ takes the value 1 for the vertices of $C_{5}$ and the value 0 for the vertices of $K_{4}$.

\section{REFERENCES}

[1] Allan, R.B. and Laskar, R.C. - On domination, independent domination numbers of a graph. Discrete Math., 23 (1978), 73 - 76.

[2] Cockayne, E.J. and Hedetniemi, S.T. - Towards a theory 261. of domination in graphs. Networks, 7 (1977), 247

[3] Cockayne, C.J., Dawes, R.M. and Hedetniemi, S.T- Total domination in graphs, Networks, 10 (1980), $211-219$.

[4] Cockayne, E.J., Mynhardt, C.M. and Yu, B.- Total dominating functions in trees: Minimality and Convexity, Journal of Graph Theory, 19(1995), 83-92.

[5] Frucht, R. and Harary, F. - On the corona of Two Graphs, Aequationes Mathematicae, Volume 4, Issue 3 (1970), $322-325$.

[6] Haynes, T.W., Hedetniemi, S.T. and Slater, P.J. Domination in Graphs: Advanced Topics, Marcel Dekker, Inc., New York, (1998).

[7] Haynes, T.W., Hedetniemi, S.T. and Slater, P.J. Fundamentals of domination in graphs, Marcel Dekker, Inc., New York , (1998).

[8] Jeelani Begum, S. - Some studies on dominating functions of Quadratic Residue Cayley Graphs, Ph. D. thesis, Sri Padmavathi Mahila Visvavidyalayam, Tirupati, Andhra Pradesh, India, (2011).

[9] Siva Parvathi, M - Some studies on dominating functions of corona product graphs, $\mathrm{Ph} . \mathrm{D}$ thesis, Sri Padmavati Mahila Visvavidyalayam, Tirupati, Andhra Pradesh, India, (2013).

[10] Siva Parvathi, M and Maheswari, B . - Minimal Dominating Functions of Corona Product Graph of a Cycle with a Complete Graph - International Journal of 
Computer Engineering \& Technology, Volume 4, Issue 4 (2013), $248-256$.

[11] Siva Parvathi, M and Maheswari, B. - Some variations of Y-Dominating Functions of Corona Product Graph of a Cycle with a Complete Graph - International Journal of Computer Applications, Volume 81, Issue 1 (2013),
$16-21$

[12] Siva Parvathi, M and Maheswari, B. - Some variations of Total Y-Dominating Functions of Corona Product Graph of a Cycle with a Complete Graph - Fire Journal of Science and Technology (accepted). 